\title{
DUNE as the Next-Generation Solar Neutrino Experiment
}

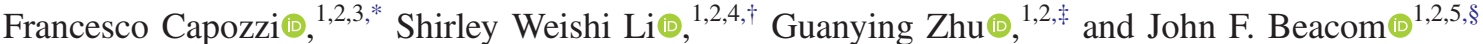 \\ ${ }^{1}$ Center for Cosmology and AstroParticle Physics (CCAPP), Ohio State University, Columbus, Ohio 43210, USA \\ ${ }^{2}$ Department of Physics, Ohio State University, Columbus, Ohio 43210, USA \\ ${ }^{3}$ Max-Planck-Institut für Physik (Werner-Heisenberg-Institut), 80805 München, Germany \\ ${ }^{4}$ SLAC National Accelerator Laboratory, Menlo Park, California 94025, USA \\ ${ }^{5}$ Department of Astronomy, Ohio State University, Columbus, Ohio 43210, USA
}

(Received 4 September 2018; published 27 September 2019)

\begin{abstract}
We show that the Deep Underground Neutrino Experiment (DUNE), with significant but feasible new efforts, has the potential to deliver world-leading results in solar neutrinos. With a $100 \mathrm{kton}-\mathrm{yr}$ exposure, DUNE could detect $\gtrsim 10^{5}$ signal events above $5 \mathrm{MeV}$ electron energy. Separate precision measurements of neutrino-mixing parameters and the ${ }^{8} \mathrm{~B}$ flux could be made using two detection channels $\left(\nu_{e}+{ }^{40} \mathrm{Ar}\right.$ and $\left.\nu_{e, \mu, \tau}+e^{-}\right)$and the day-night effect $(>10 \sigma)$. New particle physics may be revealed through the comparison of solar neutrinos (with matter effects) and reactor neutrinos (without), which is discrepant by $\sim 2 \sigma$ (and

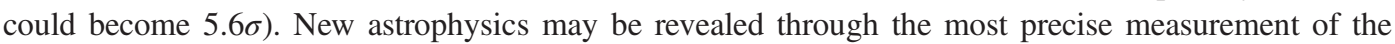
${ }^{8} \mathrm{~B}$ flux (to $2.5 \%$ ) and the first detection of the hep flux (to $11 \%$ ). DUNE is required: No other experiment, even proposed, has been shown capable of fully realizing these discovery opportunities.
\end{abstract}

DOI: 10.1103/PhysRevLett.123.131803

Introduction.-Tremendous scientific opportunities remain in solar neutrinos. What are the particle properties of neutrinos? What are the nuclear processes that power our Sun and other stars? Although the basics are known [1-6], there are multiple unknowns and discrepancies. To progress, we need precise measurements of all neutrino-producing processes, plus ways to isolate new physics from new astrophysics. Here we focus on high energies $(>5 \mathrm{MeV}$ electron energy).

For particle physics, the primary opportunity is to test for new physics through a precision comparison of neutrinomixing parameters [7-14] measured in solar versus reactor experiments. Figures 1 and 2 preview this. There is a $\sim 2 \sigma$ discrepancy for $\Delta m_{21}^{2}[6,15-17]$. The reactor measurement will soon be greatly improved by the Jiangmen Underground Neutrino Observatory (JUNO) experiment [18], but testing new physics depends on improving the solar measurement, too. The contrast in physical conditions is striking: neutrinos versus antineutrinos, matter versus vacuum mixing, plus a much larger distance, giving sensitivity to $C P T$ violation $[7,8]$, nonstandard neutrino interactions [9,19], neutrino decay [20,21], and more.

For astrophysics, the primary opportunity is to make an independent precise measurement of the ${ }^{8} \mathrm{~B}$ flux, which is

Published by the American Physical Society under the terms of the Creative Commons Attribution 4.0 International license. Further distribution of this work must maintain attribution to the author(s) and the published article's title, journal citation, and DOI. Funded by SCOAP . extremely sensitive to the solar core temperature $\left(\sim T_{c}^{25}\right.$ [23]) and which is an important ingredient for resolving the solar-metallicity discrepancy (requiring also progress on the ${ }^{7} \mathrm{Be}$ and CNO fluxes) [24-28]. Discovery of the hep flux [24-30], the highest-energy neutrino process, would probe physical conditions far from the solar center while still having large matter effects.

How can these opportunities be realized, especially simultaneously? This requires a new multi-10-kton-scale experiment, plus breakthroughs in detection strategy.

We propose that the Deep Underground Neutrino Experiment (DUNE) - intended to make transformative studies of $\mathrm{GeV}$ long-baseline neutrino mixing, proton decay, and supernova neutrino bursts [31-33] - has the potential to do the same for solar neutrinos. The budgeted plans for DUNE provide a large active volume, a huge overburden, and excellent technical capabilities, including at $\mathrm{MeV}$ energies (for supernovae) [31-33]. For solar neutrinos, DUNE would need new investments, detailed below, that would also enhance its planned programs. Building on prior work on solar neutrino detection in liquid argon [34-37], our Letter goes much further.

We review the challenges in solar neutrinos, outline our proposed strategy for DUNE, calculate the signals and backgrounds, calculate the physics reach, define technical requirements, and conclude. To show what DUNE could achieve, we calculate our main results under optimistic but feasible assumptions; we also discuss the impact of varying these assumptions. In Supplemental Material [38] and a separate paper on backgrounds [39], we provide further details. Further technical studies will be needed. 


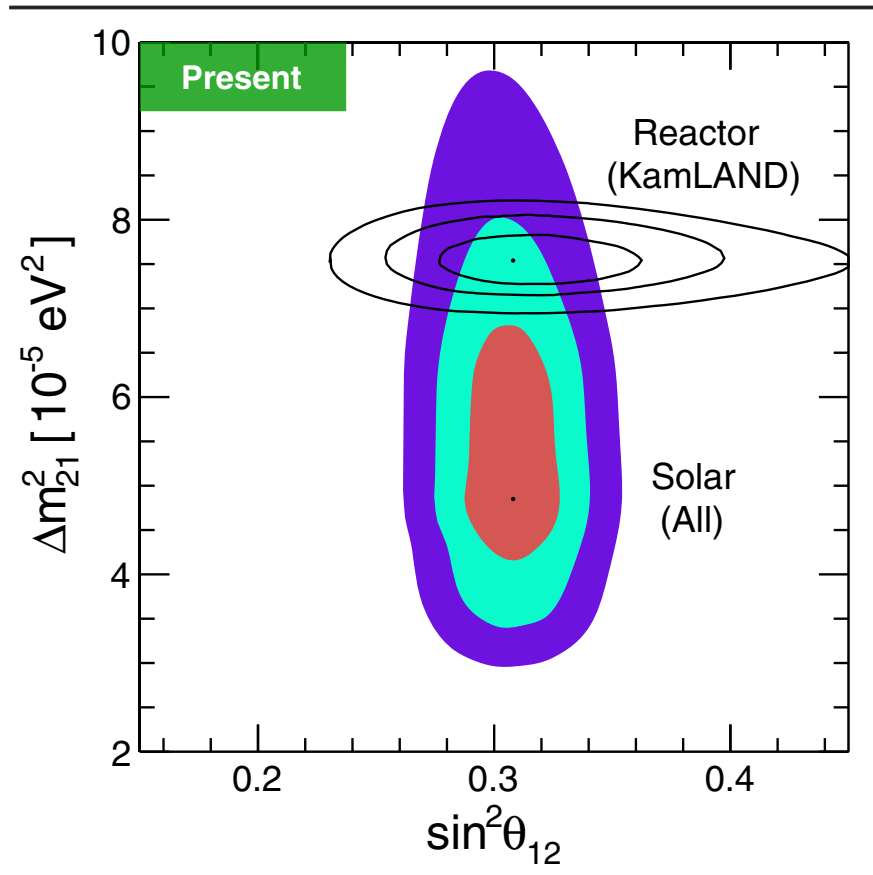

FIG. 1. Present measurements $(1,2$, and $3 \sigma)$ of neutrino mixing with solar [1-6] and reactor [15] neutrinos.

Solar neutrinos: Status and obstacles.-The fundamental challenge in solar neutrinos is disentangling neutrinomixing effects and source properties. Super-Kamiokande (Super-K) and Sudbury Neutrino Observatory (SNO) measurements of ${ }^{8} \mathrm{~B}$ neutrinos dominate the precision of solar determinations of $\sin ^{2} \theta_{12}$ and $\Delta m_{21}^{2}$, as well as $\phi\left({ }^{8} \mathrm{~B}\right)$, the total ${ }^{8} \mathrm{~B}$ flux $[5,6,12,40,41]$. The hep flux, $\phi($ hep $) \sim 10^{-3} \phi\left({ }^{8} \mathrm{~B}\right)$, has not been detected $[42,43]$.

Super-K and SNO measurements are consistent with an energy-independent $\nu_{e}$ survival probability $P_{e e} \simeq \sin ^{2} \theta_{12}$; the lack of an observed upturn in $P_{e e}$ at low energies sets a weak upper limit on $\Delta m_{21}^{2}[5,6]$. Within the theoretical framework of matter-affected neutrino mixing [44-49], these results are consistent with lower-energy solar neutrino data [1-4]. Two other results were key.

(1) SNO separately measured $\phi\left({ }^{8} \mathrm{~B}\right)$ and $\sin ^{2} \theta_{12}$ using two channels: $\nu_{e, \mu, \tau}+d \rightarrow \nu_{e, \mu, \tau}+p+n$, which is equally sensitive to all active flavors and, hence, measures the total flux, and $\nu_{e}+d \rightarrow e^{-}+p+p$, from which they can then extract the mixing angle. Progress on $\sin ^{2} \theta_{12}$ is limited primarily by SNO's final precision for $\phi\left({ }^{8} \mathrm{~B}\right)$ of $\simeq 4 \%(\simeq 3 \%$ statistical) and partially by the $1.7 \%$ systematic uncertainty on the elastic-scattering channel in Super-K $[5,6,12,40]$.

(2) Super-K best constrains $\Delta m_{21}^{2}$ by measuring the daynight flux asymmetry (at $\simeq 3 \sigma$ ) [6] with the $\nu_{e, \mu, \tau}+e^{-} \rightarrow$ $\nu_{e, \mu, \tau}+e^{-}$channel, where $P_{e e}$ at night is increased by several percent due to the matter effect in Earth [49-52]. Progress is limited by the slow increase in statistics after 20 years of exposure.

Unique advantages of DUNE.-DUNE will be in the Homestake mine in South Dakota (4300 m.w.e.). Each

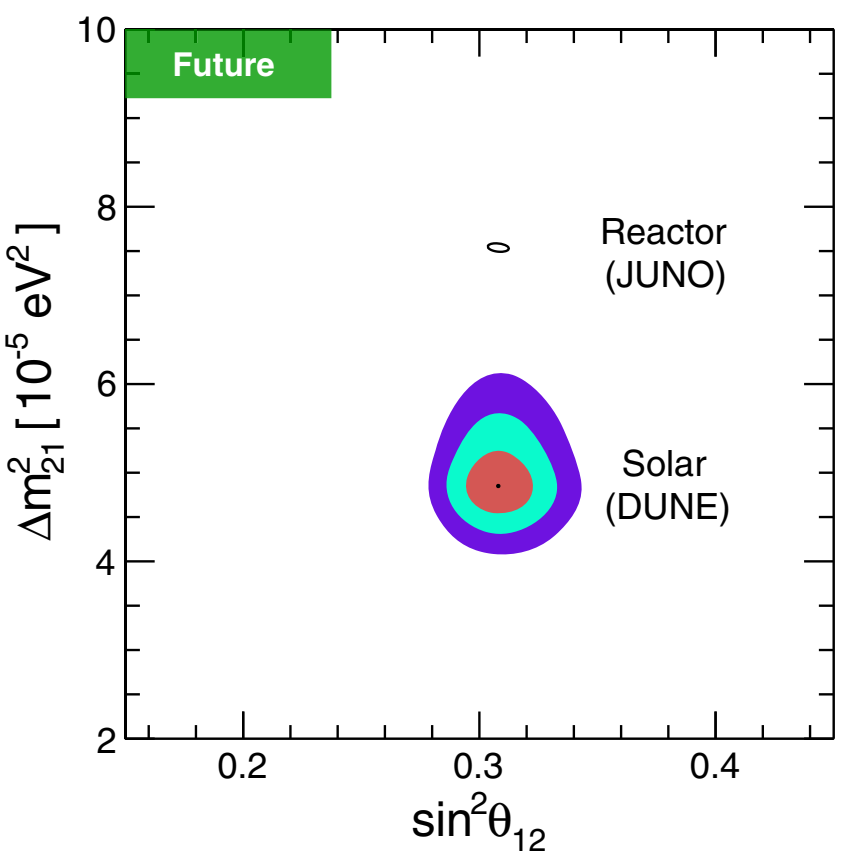

FIG. 2. Future precision of neutrino mixing with solar (DUNE alone; 1, 2, and $3 \sigma$ ) and reactor (JUNO alone; $3 \sigma$ [18,22]) neutrinos, using present best-fit points and $100 \mathrm{kton}-\mathrm{yr}$ for each.

of two liquid-argon (LAr) modules (eventually four) will have a fiducial mass of $10 \mathrm{kton}$, surrounded by $\sim 1 \mathrm{~m} \mathrm{LAr}$ shielding (details depend on single or dual phase). Readout is by the time-projection technique-here, drifting charge deposited in the volume onto wire planes at the boundaries-plus prompt detection of scintillation light [31-33,53].

DUNE can simultaneously measure neutrino-mixing parameters and solar neutrino fluxes. Here, we first state our underlying ideas and simple estimates.

(a) The degeneracy between $\sin ^{2} \theta_{12}$ and $\phi\left({ }^{8} \mathrm{~B}\right)$ can be broken using two detection channels:

$$
\nu_{e}+{ }^{40} \mathrm{Ar} \rightarrow e^{-}+{ }^{40} \mathrm{~K}^{*},
$$

where the rate $R_{\mathrm{Ar}} \propto \phi\left({ }^{8} \mathrm{~B}\right) \sin ^{2} \theta_{12}$, and

$$
\nu_{e, \mu, \tau}+e^{-} \rightarrow \nu_{e, \mu, \tau}+e^{-}
$$

where $R_{e} \propto \phi\left({ }^{8} \mathrm{~B}\right)\left(\sin ^{2} \theta_{12}+\frac{1}{6} \cos ^{2} \theta_{12}\right)$. These channels can be adequately separated with a crude angular cut. Though the dependence on the $\nu_{\mu, \tau}$ content is weak, DUNE can improve on SNO due to its huge statistics. Figure 3 illustrates this.

(b) $\Delta m_{21}^{2}$ can be isolated through the day-night flux asymmetry, $A_{D-N}=(D-N) / \frac{1}{2}(D+N)$, which scales as $\propto E_{\nu} / \Delta m_{21}^{2}$. For the solar $\Delta m_{21}^{2}$, an exposure of 100 kton-yr, and using only events above $6 \mathrm{MeV}$ electron energy (effective threshold; see below) and outside the 


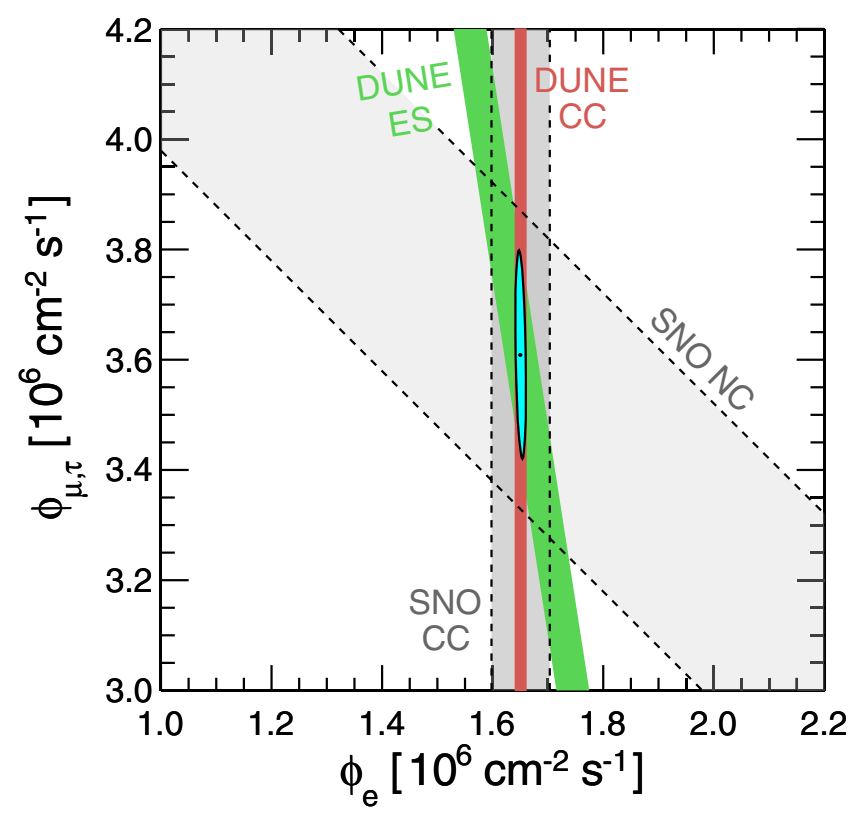

FIG. 3. Estimated precision of the $\nu_{e}$ and $\nu_{\mu, \tau}$ content of the ${ }^{8} \mathrm{~B}$ flux, present (SNO [5,54]) and future (DUNE), with the ellipse for DUNE alone. Based on a simplified analysis, with only statistical uncertainties $(1 \sigma)$ but assuming 2 d.o.f., and with SNO fluxes slightly rescaled to match their global-fit ${ }^{8} \mathrm{~B}$ flux. Note the small axis ranges. Full analysis in the text.

forward cone, we expect $D=3.04 \times 10^{4}$ and $N=3.29 \times$ $10^{4}$ signal $\nu_{e}+{ }^{40} \mathrm{Ar}$ events, along with $0.83 \times 10^{4}$ background events in total (as detailed below, and conservatively including $\nu_{e, \mu, \tau}+e^{-}$events). Considering for now only statistical uncertainties, we expect $A_{D-N} \simeq-(7.9 \pm 0.8) \%$ $(\sim 10 \sigma)$. DUNE can improve on Super-K, because the $\nu_{e}+{ }^{40} \mathrm{Ar}$ channel has a larger cross section, emphasizes larger neutrino energies, and has a tighter relation between neutrino and electron energy.

Solar neutrinos in DUNE.-The MeV-range capabilities of DUNE [31-33] are designed for detecting supernova neutrinos. Above $5 \mathrm{MeV}$, we assume electrons can be detected with high efficiency and 7\% energy-independent energy resolution [33]. For solar signals, electrons lose energy dominantly by ionization, as the critical energy of LAr is $32 \mathrm{MeV}$ [55-57]. The angular resolution of DUNE is uncertain; we adopt $25^{\circ}$, based on ICARUS simulations [35]. Below, we discuss the impact of different assumptions.

We use neutrino spectra from Refs. [58,59] and radial distributions from the BS05(OP) model of Refs. [25,26]. As nominal fluxes, we use $\phi\left({ }^{8} \mathrm{~B}\right)=5.25 \times 10^{6} \mathrm{~cm}^{-2} \mathrm{~s}^{-1}$ (4\% uncertainty, from SNO [5]) and $\phi($ hep $)=$ $8.25 \times 10^{3} \mathrm{~cm}^{-2} \mathrm{~s}^{-1} \quad(30 \%$ uncertainty, from theory $[28,30])$. The end point energies are $\simeq 15$ and $\simeq 19 \mathrm{MeV}$.

For the charged-current (CC) channel $\nu_{e}+{ }^{40} \mathrm{Ar}$ [60-74], the ground state threshold is $Q_{\text {g.s. }}=1.5 \mathrm{MeV}$ [75], but this transition is forbidden. The cross section is dominated by transitions to nuclear excited states in ${ }^{40} \mathrm{~K}^{*}$ (a superallowed Fermi transition with $\Delta E_{i}=4.4 \mathrm{MeV}$, plus several Gamow-Teller transitions), which promptly produce gamma rays by nuclear deexcitation. Because of these nuclear thresholds, DUNE is most sensitive to $E_{\nu} \gtrsim$ $9 \mathrm{MeV}$. We define the detectable energy of an event as the electron kinetic energy $T_{e}$, given by $T_{e}=E_{\nu}-Q$, where $Q=Q_{\text {g.s. }}+\Delta E_{i}$, conservatively neglecting the detectability of the $\Delta E_{i}$ in gamma rays (if these gamma rays were detectable, that would dramatically improve event identification, background rejection, energy reconstruction, and sensitivity). The electrons are emitted near isotropically. Details, including cross section uncertainties, are discussed below and in Supplemental Material [38].

For the elastic-scattering (ES) channel $\nu_{e, \mu, \tau}+e^{-}$, there is no threshold, and the cross section is known with subpercent precision [76]. All flavors participate, but the sensitivity to the $\nu_{\mu, \tau}$ content is reduced, as these have only neutral-current couplings. DUNE is sensitive to $E_{\nu} \gtrsim 5 \mathrm{MeV}$, though the broad differential cross section effectively raises that. The direction of the scattered electron is well correlated to the neutrino direction, with a maximum scattering angle of about $20^{\circ}$. We adequately separate $\nu_{e, \mu, \tau}+e^{-}$and $\nu_{e}+{ }^{40} \mathrm{Ar}$ events by defining a forward cone of half-angle $40^{\circ}$, maximizing the signal to background ratios for both event categories in the cone away from the Sun and its complement. Inside the cone, which includes $81 \%$ of $\nu_{e, \mu, \tau}+e^{-}$events [35], they dominate; outside the cone, which includes $88 \%$ of $\nu_{e}+{ }^{40} \mathrm{Ar}$ events, they dominate.

In principle, DUNE could use the neutral-current (NC) channel $\nu_{e, \mu, \tau}+{ }^{40} \mathrm{Ar} \rightarrow \nu_{e, \mu, \tau}+{ }^{40} \mathrm{Ar}^{*}$, where the final state is detected through nuclear gamma rays $[68,70,77]$. We treat this as a background because the cross section seems small. If not, this could be an important new signal.

Backgrounds must be mitigated with standard MeVdetector techniques: defining a fiducial volume, removing $\mathrm{U}$ and $\mathrm{Th}$ from liquids and $\mathrm{Rn}$ from air, selecting low-background materials, applying dead time after high-energy events, etc. [1-6,15,18,78,79]. Three important backgrounds will remain (details in Supplemental Material [38] and Ref. [39]). First are neutron captures on ${ }^{40} \mathrm{Ar}$, releasing a total of $6.1 \mathrm{MeV}$ in several gamma rays [80-82]; these Compton scatter or pair-produce electrons. These neutrons, most less than a few $\mathrm{MeV}$, are dominantly produced by $(\alpha, n)$ interactions in the rock following U- and Th-chain decays [35,83,84]; muon-induced neutrons are relatively negligible [39]. Once neutrons enter the detector, they fill the volume, due to their small cross section on argon. We assume a hermetic, passive water (or oil or plastic) shield of $40-\mathrm{cm}$ thickness, reducing this background by $\sim 4 \times 10^{3}$. Below, we show that even no shielding is acceptable. Second, neutral-current $\nu_{e, \mu, \tau}+{ }^{40} \mathrm{Ar}$ events cause a peak near $9 \mathrm{MeV}$ [77]. Third, at the highest energies, are beta-decaying radioactivities 

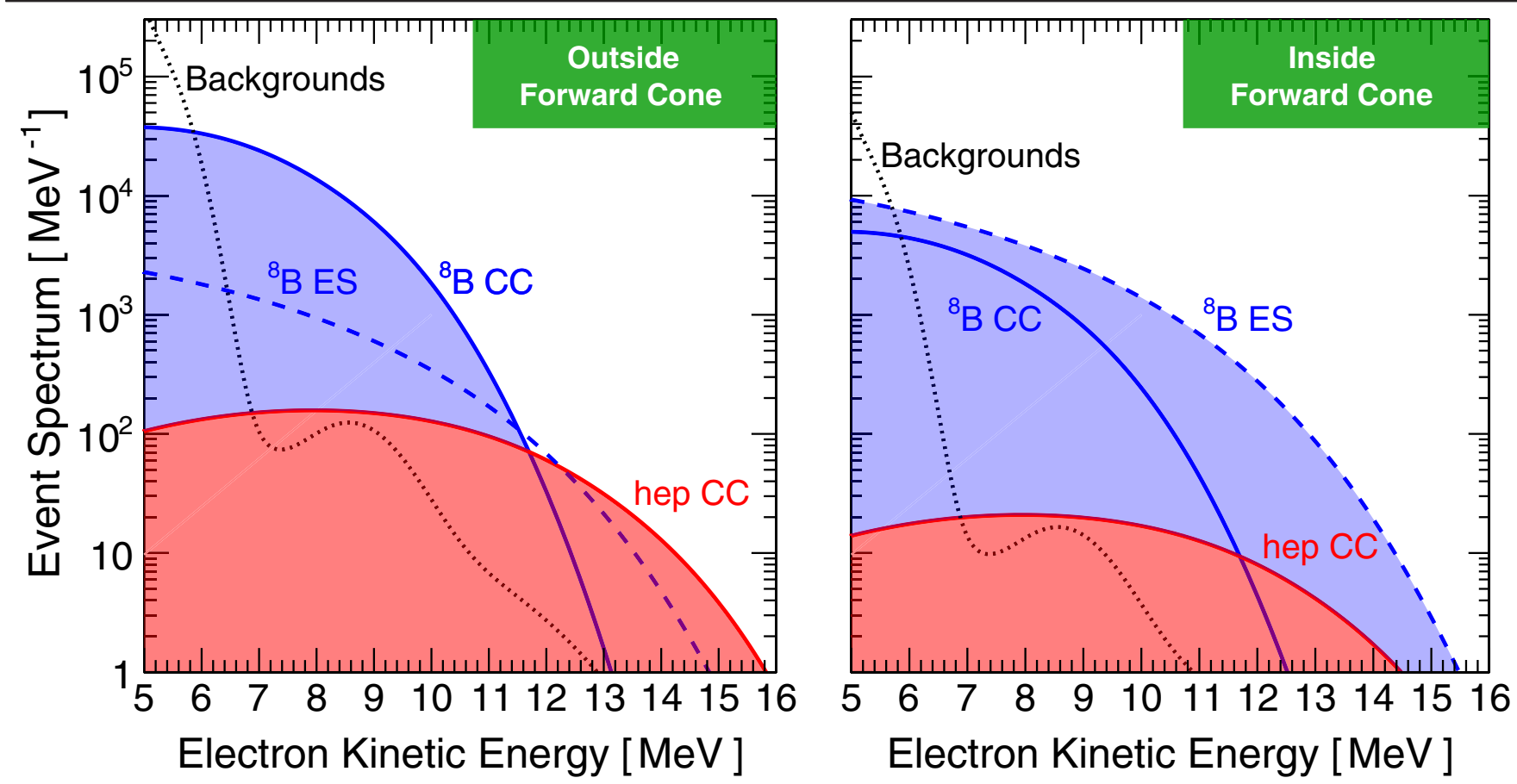

FIG. 4. Predicted solar neutrino signals and backgrounds in DUNE for $100 \mathrm{kton}-\mathrm{yr}$, using a forward cone of half-angle $40^{\circ}$ and (here only) combining day and night data. We include all factors discussed in the text.

induced by muons [39,85-87], for which we apply simple cuts. The pileup rates from these and other backgrounds (e.g., ${ }^{39} \mathrm{Ar}$ and ${ }^{42} \mathrm{Ar}$ decays) are negligible [39].

Figure 4 shows the solar neutrino signal and background spectra in DUNE. Our calculations include three-flavor neutrino mixing [44-51], realistic detection effects (differential cross sections [69,88-90], energy smearing, angular cuts [35], background reduction [75,91-104]), and a 100 kton-yr exposure.

For ${ }^{8} \mathrm{~B}$ events, the two channels are well separated and have superb yields above $5 \mathrm{MeV}$ electron energy. For $\nu_{e}+{ }^{40} \mathrm{Ar}$, there are $9.9 \times 10^{4}$ events outside the forward cone. For $\nu_{e, \mu, \tau}+e^{-}$, there are $2.6 \times 10^{4}$ events inside the forward cone. This channel provides better sensitivity to lower-energy neutrinos and the only sensitivity to $\nu_{\mu, \tau}$. For hep events, the $\nu_{e}+{ }^{40} \mathrm{Ar}$ channel allows a clear separation at high electron energies, with 150 events above $11 \mathrm{MeV}$ (the small $\nu_{e, \mu, \tau}+e^{-}$channel is not shown).

DUNE physics reach.-DUNE can significantly improve the precision of solar neutrino observables. We jointly fit (without priors) four parameters: $\sin ^{2} \theta_{12}, \Delta m_{21}^{2}, \phi\left({ }^{8} \mathrm{~B}\right)$, and $\phi($ hep $)$. When reporting projected uncertainties for $n$ parameters, we marginalize over the others, adopting $\Delta \chi^{2}$ confidence levels for $n$ d.o.f. We assume that new physics affecting solar neutrinos is reflected in mixing-parameter values that differ from the reactor values. We use the theoretically expected counts for signals and backgrounds, with Poisson uncertainties (below, we discuss systematics). We partition data into bins of energy, bins of Earth zenith angle (night only), and outside or inside the forward cone (as in Fig. 4). We use the total electron spectra, assuming only statistical separation of the components and not requiring neutrino-energy reconstruction.

Figure 2 shows the projected precision for DUNE's measurement of neutrino-mixing parameters, assuming the solar best-fit values $\left(\sin ^{2} \theta_{12}=0.308\right.$ and $\Delta m_{21}^{2}=$ $4.85 \times 10^{-5} \mathrm{eV}^{2}$ [6]). The uncertainties are $3.0 \%$ and $5.9 \%$, a factor of $\simeq 1.5$ and $\simeq 3$ better than from all solar experiments to date, respectively, as shown in Fig. 1. The sensitivity to $\Delta m_{21}^{2}$ comes primarily from the day-night effect $(10.4 \sigma)$. The ${ }^{8} \mathrm{~B}$ flux (marginalizing over other parameters) can be measured to $2.5 \%$, a factor of $\simeq 1.6$ better than from SNO. DUNE can make a robust first detection of hep neutrinos, with a precision of $11 \%$, a factor of $\simeq 3$ better than the current theoretical uncertainty.

Going forward.-New investments are needed to enhance the $\mathrm{MeV}$ capabilities of DUNE. At the trigger level, this includes enhancing data acquisition, storage, and processing for a steady rate of $\mathrm{MeV}$ events. Calibration at $\mathrm{MeV}$ energies across the detector volume will be crucial to controlling systematics. An enhanced light-detection system would enhance $\mathrm{MeV}$ detection.

Backgrounds must be controlled, and the biggest concern, after standard cuts $[1-6,15,18,78,79]$, is neutron captures [39]. We see three possible strategies.

$1.40 \mathrm{~cm}$ of shielding, as assumed above.-This allows a low threshold $(\simeq 5.8 \mathrm{MeV})$ to test for shape distortions in the spectrum and to enhance particle-identification techniques.

2. Additional run time.-With less or no shielding, the effective analysis threshold would be higher. This can be 
compensated by a larger exposure than 100 kton-yr. With $30,20,10$, or $0 \mathrm{~cm}$ of shielding, the effective analysis threshold is $\simeq 6.2,6.5,6.9$, or $7.2 \mathrm{MeV}$, and the exposure needed for comparable results increases at most of a factor of $\sim 2$.

3. Better particle-identification techniques.-We assume that neutrino-interaction events and neutroncapture events with the same electron energy are indistinguishable. This is too conservative, because $\nu_{e, \mu, \tau}+e^{-}$, $\nu_{e}+{ }^{40} \mathrm{Ar}$, and neutron-capture events would have one electron, an electron with $\gamma$ rays, and multiple $\gamma$ rays, respectively. For $\gamma$ rays, the radiation length is $14 \mathrm{~cm}$ and Compton scattering dominates [55], so these event classes should be distinct.

The impact of varying the assumptions made above is detailed in Supplemental Material [38]. Here, we summarize some key points. The sensitivity to $\Delta m_{21}^{2}$ is very robust, because it is determined from the day-night effect, which cancels many inputs. The uncertainty on the $\nu_{e}+{ }^{40} \mathrm{Ar}$ cross section (presently 10\%) and the detector systematics should be reduced to $1 \%$. Without this, DUNE alone cannot break the degeneracy between $\sin ^{2} \theta_{12}$ and $\phi\left({ }^{8} \mathrm{~B}\right)$, though its measurement of $\Delta m_{21}^{2}$ would be unaffected and its measurement of $\phi($ hep) be only modestly affected. Importantly, the intended precision of $\sin ^{2} \theta_{12}$ and $\phi\left({ }^{8} \mathrm{~B}\right)$ could be largely restored by combining with existing solar data. If the energy resolution is $20 \%$, the intended precision of $\phi($ hep) would degrade to $18 \%$, though that of $\sin ^{2} \theta_{12}, \phi\left({ }^{8} \mathrm{~B}\right)$, and $\Delta m_{21}^{2}$ could be largely restored by increasing exposure by $\sim 2$. DUNE's sensitivity would be lost if the energy resolution is poor and backgrounds are not reduced.

Concluding perspectives.-This is the first study to detail how DUNE, with a new, challenging but feasible solar neutrino program, would open substantial discovery space in both particle physics and astrophysics. With DUNE's precision measurements of $\sin ^{2} \theta_{12}$ and $\Delta m_{21}^{2}$, the comparison to JUNO's may reveal new particle physics of neutrinos. Simultaneously, with DUNE's precision measurements of $\phi\left({ }^{8} \mathrm{~B}\right)$ and $\phi($ hep$)$, it may reveal new astrophysics. Further studies are needed to evaluate our proposal and to optimize its sensitivity.

No other planned experiment has been shown potentially capable of meeting all of these goals. Of proposed experiments, Hyper-Kamiokande (Hyper-K) $[105,106]$ stands out, and it can nicely complement DUNE. Hyper-K would have only one channel, $\nu_{e, \mu, \tau}+e^{-}$, but huge statistics. DUNE and Hyper-K would measure $\Delta m_{21}^{2}$ (from the daynight asymmetry) comparably well. Hyper-K would have a significant advantage on measuring the upturn in the $\nu_{e}$ survival probability. DUNE would measure $\phi($ hep) much better. Their combined impact would be significantly enhanced by new experiments for low-energy solar neutrinos $[36,107,108]$.
Solar neutrino studies, begun long ago, are not done. DUNE can lead the next generation of discoveries.

For helpful discussions, we are grateful to Manojeet Bhattacharya, Ed Blucher, Steve Brice, Mauricio Bustamante, Gustavo Cancelo, David Caratelli, Flavio Cavanna, Alex Friedland, Dick Furnstahl, Cristiano Galbiati, Alejandro Garcia, Mathew Graham, Jeff Hartnell, Alex Himmel, Brennan Jordan, Josh Klein, Pierre Lasorak, Eligio Lisi, Joe Lykken, Kenny Ng, Gabriel Orebi Gann, Ornella Palamara, Wendy Panero, Stephen Parke, Ryan Patterson, Simon Peeters, Georg Raffelt, Juergen Reichenbacher, Elizabeth Ricard-McCutchan, David Schmitz, Kate Scholberg, Michael Smy, Bob Svoboda, Mark Vagins, Francesco Vissani, Petr Vogel, and Kyle Wendt. The work of all authors was supported by National Science Foundation Grants No. PHY-1404311 and No. PHY1714479 awarded to J.F. B. F. C. was later supported by Deutsche Forschungsgemeinschaft Grants No. EXC 153 and No. SFB 1258, as well as by European Union Grant No. H2020-MSCA-ITN-2015/674896. S. W. L. was also supported by an Ohio State Presidential Fellowship and later at SLAC by the Department of Energy under Contract No. DE-AC02-76SF00515.

We speak for ourselves as theorists, not on behalf of the DUNE Collaboration. This work is based on our ideas, our calculations, and publicly available information.

*capozzi@mpp.mpg.de

†shirleyl@slac.stanford.edu

zhu.1475@osu.edu

beacom.7@osu.edu

[1] B. T. Cleveland, T. Daily, R. Davis, Jr., J. R. Distel, K. Lande, C. K. Lee, P.S. Wildenhain, and J. Ullman, Astrophys. J. 496, 505 (1998).

[2] M. Altmann et al. (GNO Collaboration), Phys. Lett. B 616, 174 (2005).

[3] J. N. Abdurashitov, V. N. Gavrin, V. V. Gorbachev, P. P. Gurkina, T. V. Ibragimova, A. V. Kalikhov, N. G. Khairnasov, T. V. Knodel, I. N. Mirmov, A. A. Shikhin et al. (SAGE Collaboration), Phys. Rev. C 80, 015807 (2009).

[4] G. Bellini et al. (Borexino Collaboration), Phys. Rev. Lett. 107, 141302 (2011).

[5] B. Aharmim et al. (SNO Collaboration), Phys. Rev. C 88, 025501 (2013).

[6] K. Abe et al. (Super-Kamiokande Collaboration), Phys. Rev. D 94, 052010 (2016).

[7] G. Barenboim, L. Borissov, J. D. Lykken, and A. Y. Smirnov, J. High Energy Phys. 10 (2002) 001.

[8] A. de Gouvea and C. Peña-Garay, Phys. Rev. D 71, 093002 (2005).

[9] A. Friedland, C. Lunardini, and C. Peña-Garay, Phys. Lett. B 594, 347 (2004).

[10] M. Cirelli, M. C. González-García, and C. Peña-Garay, Nucl. Phys. B719, 219 (2005).

[11] A. Palazzo, Phys. Rev. D 83, 113013 (2011). 
[12] M. Maltoni and A. Yu. Smirnov, Eur. Phys. J. A 52, 87 (2016).

[13] F. Capozzi, I. M. Shoemaker, and L. Vecchi, J. Cosmol. Astropart. Phys. 07 (2017) 021.

[14] S.-H. Seo and S. J. Parke, Phys. Rev. D 99, 033012 (2019).

[15] A. Gando, Y. Gando, H. Hanakago, H. Ikeda, K. Inoue, K. Ishidoshiro, H. Ishikawa, M. Koga, R. Matsuda, S. Matsuda et al. (KamLAND Collaboration), Phys. Rev. D 88, 033001 (2013).

[16] I. Esteban, M. C. González-García, M. Maltoni, I. Martínez-Soler, and T. Schwetz, J. High Energy Phys. 01 (2017) 087.

[17] F. Capozzi, E. Lisi, A. Marrone, and A. Palazzo, Prog. Part. Nucl. Phys. 102, 48 (2018).

[18] F. An et al. (JUNO Collaboration), J. Phys. G 43, 030401 (2016).

[19] J. Liao, D. Marfatia, and K. Whisnant, Phys. Lett. B 771, 247 (2017).

[20] J. F. Beacom and N. F. Bell, Phys. Rev. D 65, 113009 (2002).

[21] J. M. Berryman, A. de Gouvea, and D. Hernandez, Phys. Rev. D 92, 073003 (2015).

[22] F. Capozzi, E. Lisi, and A. Marrone, Phys. Rev. D 92, 093011 (2015).

[23] J. N. Bahcall and A. Ulmer, Phys. Rev. D 53, 4202 (1996).

[24] N. Grevesse and A. J. Sauval, Space Sci. Rev. 85, 161 (1998).

[25] J. N. Bahcall, A. M. Serenelli, and S. Basu, Astrophys. J. 621, L85 (2005).

[26] J. N. Bahcall, Software and data for solar neutrino research (2005).

[27] M. Asplund, N. Grevesse, A. J. Sauval, and P. Scott, Annu. Rev. Astron. Astrophys. 47, 481 (2009).

[28] N. Vinyoles, A. M. Serenelli, F. L. Villante, S. Basu, J. Bergström, M. C. González-García, M. Maltoni, C. Peña-Garay, and N. Song, Astrophys. J. 835, 202 (2017).

[29] L. E. Marcucci, R. Schiavilla, M. Viviani, A. Kievsky, S. Rosati, and J. F. Beacom, Phys. Rev. C 63, 015801 (2000).

[30] T. S. Park, L. E. Marcucci, R. Schiavilla, M. Viviani, A. Kievsky, S. Rosati, K. Kubodera, D. P. Min, and M. Rho, Phys. Rev. C 67, 055206 (2003).

[31] R. Acciarri et al. (DUNE Collaboration), arXiv:1512.06148.

[32] J. Strait et al. (DUNE Collaboration), arXiv:1601.05823.

[33] R. Acciarri et al. (DUNE Collaboration), arXiv:1601.02984.

[34] J. N. Bahcall, M. Baldo-Ceolin, D. B. Cline, and C. Rubbia, Phys. Lett. B 178, 324 (1986).

[35] F. Arneodo et al., Nucl. Instrum. Methods Phys. Res., Sect. A 455, 376 (2000).

[36] D. Franco et al., J. Cosmol. Astropart. Phys. 08 (2016) 017.

[37] A. N. Ioannisian, A. Y. Smirnov, and D. Wyler, Phys. Rev. D 96, 036005 (2017).

[38] See Supplemental Material at http://link.aps.org/ supplemental/10.1103/PhysRevLett.123.131803 for a thorough discussion on charged-current neutrino interactions, the calculation of backgrounds, and the statistical analysis with different input choices.

[39] G. Zhu, S. W. Li, and J. F. Beacom, Phys. Rev. C 99, 055810 (2019).
[40] G. L. Fogli, E. Lisi, A. Marrone, and A. Palazzo, Prog. Part. Nucl. Phys. 57, 742 (2006).

[41] B. Aharmim et al. (SNO Collaboration), Phys. Rev. C 72, 055502 (2005).

[42] J. Hosaka et al. (Super-Kamiokande Collaboration), Phys. Rev. D 73, 112001 (2006).

[43] A. T. Mastbaum, Constraining the hep solar neutrino and diffuse Supernova neutrino background fluxes with the Sudbury neutrino observatory, $\mathrm{Ph}$. D. thesis, University of Pennsylvania, 2016.

[44] L. Wolfenstein, Phys. Rev. D 17, 2369 (1978).

[45] S. P. Mikheev and A. Yu. Smirnov, Yad. Fiz. 42, 1441 (1985) [Sov. J. Nucl. Phys. 42, 913 (1985)].

[46] S. P. Mikheev and A. Yu. Smirnov, Nuovo Cimento C 9, 17 (1986).

[47] W. C. Haxton, Phys. Rev. Lett. 57, 1271 (1986).

[48] S. J. Parke, Phys. Rev. Lett. 57, 1275 (1986).

[49] C. Giunti and C. W. Kim, Fundamentals of Neutrino Physics and Astrophysics (Oxford University Press, Oxford, 2007).

[50] E. Lisi and D. Montanino, Phys. Rev. D 56, 1792 (1997).

[51] M. Maris and S. T. Petcov, Phys. Rev. D 56, 7444 (1997).

[52] W. C. Forsythe, E. J. Rykiel, Jr., R. S. Stahl, H.-i. Wu, and R. M. Schoolfield, Ecol. Model. 80, 87 (1995).

[53] A. Marchionni, Annu. Rev. Nucl. Part. Sci. 63, 269 (2013).

[54] B. Aharmim et al. (SNO Collaboration), Phys. Rev. C 87, 015502 (2013).

[55] C. Patrignani et al. (Particle Data Group), Chin. Phys. C 40, 100001 (2016).

[56] S. Amoruso et al. (ICARUS Collaboration), Eur. Phys. J. C 33, 233 (2004).

[57] R. Acciarri et al. (MicroBooNE Collaboration), J. Instrum. 12, P09014 (2017).

[58] W. T. Winter, S. J. Freedman, K. E. Rehm, and J. P. Schiffer, Phys. Rev. C 73, 025503 (2006).

[59] J. N. Bahcall, Phys. Rev. C 56, 3391 (1997).

[60] E. J. Konopinski, The Theory of Beta Radioactivity (Oxford University Press, Oxford, 1950).

[61] W. E. Ormand, P. M. Pizzochero, P. F. Bortignon, and R. A. Broglia, Phys. Lett. B 345, 343 (1995).

[62] M. Bhattacharya et al., Phys. Rev. C 58, 3677 (1998).

[63] J.F. Beacom and P. Vogel, Phys. Rev. D 60, 033007 (1999).

[64] P. Vogel and J.F. Beacom, Phys. Rev. D 60, 053003 (1999).

[65] J. F. Beacom and S. J. Parke, Phys. Rev. D 64, 091302(R) (2001).

[66] A. Kurylov, M. J. Ramsey-Musolf, and P. Vogel, Phys. Rev. C 67, 035502 (2003).

[67] I. Gil Botella and A. Rubbia, J. Cosmol. Astropart. Phys. 10 (2003) 009.

[68] E. Kolbe, K. Langanke, G. Martinez-Pinedo, and P. Vogel, J. Phys. G 29, 2569 (2003).

[69] M. Bhattacharya, C. D. Goodman, and A. Garcia, Phys. Rev. C 80, 055501 (2009).

[70] M.-K. Cheoun, E. Ha, and T. Kajino, Phys. Rev. C 83, 028801 (2011).

[71] T. Suzuki, M. Honma, A. B. Balantekin, T. Kajino, and S. Chiba, EPJ Web Conf. 66, 07025 (2014). 
[72] J. C. Hardy and I. S. Towner, Phys. Rev. C 91, 025501 (2015).

[73] M. Karakoç, R. G. T. Zegers, B. A. Brown, Y. Fujita, T. Adachi, I. Boztosun, H. Fujita, M. Csatlós, J. M. Deaven, C. J. Guess et al., Phys. Rev. C 89, 064313 (2014).

[74] D. Akimov et al. (COHERENT Collaboration), Science 357, 1123 (2017).

[75] National Nuclear Data Center, NuDat 2.7 (2016).

[76] J. N. Bahcall, M. Kamionkowski, and A. Sirlin, Phys. Rev. D 51, 6146 (1995).

[77] R. S. Raghavan, S. Pakvasa, and B. A. Brown, Phys. Rev. Lett. 57, 1801 (1986).

[78] Y. Takeuchi et al. (SuperKamiokade Collaboration), Phys. Lett. B 452, 418 (1999).

[79] I. Blevis et al., Nucl. Instrum. Methods Phys. Res., Sect. A 517, 139 (2004).

[80] R. Hardell and C. Beer, Phys. Scr. 1, 85 (1970).

[81] C. D. Nesaraja and E. A. McCutchan, Nucl. Data Sheets 133, 1 (2016).

[82] National Nuclear Data Center, CapGam (2013).

[83] H. Wulandari, J. Jochum, W. Rau, and F. von Feilitzsch, Astropart. Phys. 22, 313 (2004).

[84] L. de Viveiros, Optimization of signal versus background in liquid Xe detectors used for dark matter direct detection experiments, Ph.D. thesis, Brown University, 2010.

[85] S. W. Li and J. F. Beacom, Phys. Rev. C 89, 045801 (2014).

[86] S. W. Li and J.F. Beacom, Phys. Rev. D 91, 105005 (2015).

[87] S. W. Li and J.F. Beacom, Phys. Rev. D 92, 105033 (2015).

[88] H. Behrens and J. Jänecke, Numerical Tables for BetaDecay and Electron Capture (Springer, New York, 1969).

[89] G. K. Schenter and P. Vogel, Nucl. Sci. Eng. 83, 393 (1983).

[90] A. C. Hayes and P. Vogel, Annu. Rev. Nucl. Part. Sci. 66, 219 (2016).
[91] H. Rogers, in Metallogeny of Gold in the Black Hills, South Dakota (Society of Economic Geologists, 1990), p. 204.

[92] J. K. Shultis and R. E. Faw, Fundamentals of Nuclear Science and Engineering (Taylor \& Francis, London, 2002).

[93] A. Ferrari, P. R. Sala, A. Fasso, and J. Ranft, FLUKA: A multi-particle transport code (Program version 2005) (2005).

[94] G. Battistoni, S. Muraro, P. R. Sala, F. Cerutti, A. Ferrari, S. Roesler, A. Fasso, and J. Ranft, AIP Conf. Proc. 896, 31 (2007).

[95] K. T. Lesko et al., arXiv:1108.0959.

[96] Y.-D. Chan, The Low-Background Construction of Laboratories at the 4850-ft Level Davis Campus (2012).

[97] B. Ricci, F. Mantovani, M. Baldoncini, J. Esposito, L. Ludhova, and S. Zavatarelli, Proc. Sci., Neutel2013 (2013) 077.

[98] J. Heise, J. Phys. Conf. Ser. 606, 012015 (2015).

[99] S. Westerdale and P. D. Meyers, Nucl. Instrum. Methods Phys. Res., Sect. A 875, 57 (2017).

[100] J. F. Beacom et al. (Jinping Collaboration), Chin. Phys. C 41, 023002 (2017).

[101] T. K. Gaisser and M. Honda, Annu. Rev. Nucl. Part. Sci. 52, 153 (2002).

[102] K. Abe et al. (Super-Kamiokande Collaboration), Phys. Rev. D 97, 072001 (2018).

[103] J. F. Beacom, Annu. Rev. Nucl. Part. Sci. 60, 439 (2010).

[104] K. Bays et al. (Super-Kamiokande Collaboration), Phys. Rev. D 85, 052007 (2012).

[105] K. Abe et al. (Hyper-Kamiokande Collaboration), arXiv: 1109.3262.

[106] K. Abe et al. (Hyper-Kamiokande Collaboration), arXiv: 1805.04163 .

[107] S. Andringa et al. (SNO+Collaboration), Adv. High Energy Phys. 2016, 6194250 (2016).

[108] J. Aalbers et al. (DARWIN Collaboration), J. Cosmol. Astropart. Phys. 11 (2016) 017. 